\title{
The effects of two lubricating products in elderly individuals - a pilot study
}

\begin{abstract}
Objective: To evaluate two products to treat symptoms of oral dryness in frail, Elderly individuals.

Material \& methods: 22 participants were recruited for this pilot study from Karolinska Institutet, Department of Dental Medicine section for Gerodontics and Public Medical Dental Care clinic at Stockholms Sjukhem and 15 fulfilled the study. The participants used Proxident oral spray with sunflower oil for 4 weeks and then Proxident gel-based flavorless oral moisturizer for 4 weeks. Registration of current medications, general health, dental status, status of the mucosa, plaque index, gingival index, objective and subjective saliva secretion was measured and microbial samples taken at baseline and after four and eight weeks (three times). The study also included measurements of quality of life.
\end{abstract}

Results: Gingival index and MPS (mucosal-plaque score) mean values were improved during the study. Plaque index was relatively constant and no statistically significant changes were found in salivary flow. Lactobacilli increased, Streptococcus mutans were constant and Candida albicans were decreased during the study period but this was not statistically significant, only as a tendency to differences. After the use of each product for 4 weeks, questionnaires OHIP-14 (Oral Health Impact Profile) and VAS (visual analogue scales) showed that participants had less problems.

Conclusion: Proxident oral spray with sunflower oil and non-flavored moisture gel may help patients suffering from dry mouth to improve their oral mucous conditions but it requires further studies to confirm these results. The products can be recommended as comfort products.

Keywords: dry mouth, hyposalivation, moisturizing products, oral micro flora, salivary flow
Volume 8 Issue 7 - 2017

\author{
Girestam Croonquist $C^{1,3}$ Inger Wardh ${ }^{1,2}$ \\ Gundler $A^{2}$ \\ 'Academic Centre of Geriatric dentistry, Sweden \\ ${ }^{2}$ Department of Dental Medicine, Karolinska Institute, Sweden \\ ${ }^{3}$ Stockholm county Public dental care AB, Sweden
}

\begin{abstract}
Correspondence: Inger Wardh, Department of Dental Medicine, Karolinska institutet, Box 4064, I4I 04 Huddinge, Sweden, Email inger.wardh@ki.se
\end{abstract}

Received: November 24, 2017 | Published: December 01, 2017

\section{Clinical relevance}

1. Scientific rationale for study: The market offers different products to treat the symptoms of oral dryness but there is no scientific evidence for the effects.

2. Principal findings: This study shows that lubricating products could be recommended to patients with dry mouth to increase their quality of life. It may also improve their oral mucous conditions.

3. Practical implications: Lubricating products should primarily be recommended as comfort products. The patient ought to be given the opportunity to choose which product to use.

\section{Introduction}

Saliva production is important for both oral and general health Hyposalivation increases the risk of caries and other diseases, especially in elderly. 1 Saliva also plays an important role in facilitating speech and the process of swallowing. Today we have an increasing number of elderly who still have their natural teeth. Elderly people are in the high-risk group for hypo-salivation ${ }^{2}$ since they often use several different medications and the majority of these products have an anticholinergic effect with dry mouth as one of many side-effects. ${ }^{3}$ The oral health of dependent and vulnerable elderly in need of special care plays a vital role for each individual. Dry mouth can also affect the quality of $\mathrm{life}^{4}$ besides the oral and medical effects. Oral dryness also causes a change in an individual's oral microbial state. Individuals with poor oral hygiene have been found to have an increased rate of upper respiratory tract infections, such as pneumonia. ${ }^{5}$ Aspiration pneumonia is the second most common infection in elderly in nursing homes, which requires hospital treatment and has the highest mortality. ${ }^{6}$ In a study of the risk factors, it was found that a lack of oral hygiene and swallowing difficulties had a significant relationship with pneumonia incidence. ${ }^{7}$ Frail, elderly people with impaired immune systems may aspirate usually harmless oral micro-flora as candida and these results in opportunistic infections. ${ }^{6}$ In the last years lubricating products have been popular as a treatment against oral dryness. Nursing staff inform the residents about products and help them to handle the products in a pharmacy. ${ }^{8}$ The market offers different products but few of these products have been evaluated. They are not supposed to stimulate the saliva but to a certain extent replace missing mucus saliva and give comfort.

\section{Objective}

The aim of this study is to evaluate two products marketed as products that relieve dry and sensitive mouth in frail, elderly 
individuals with respect to the status of gingiva and mucosa, salivary secretion, microbial flora and oral health-related quality of life.

\section{Materials and methods}

\section{Study design}

The study had a descriptive, longitudinal, evaluating design. Figure 1 presents a flow diagram.

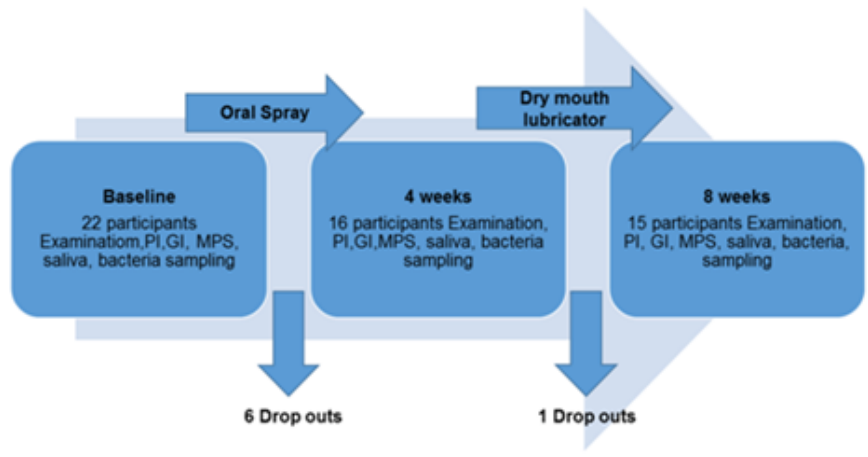

Figure I Flow chart. Baseline-drop-outs $(n=6)$ Oral spray 4 weeks-drop-outs $(n=1)$ Dry mouth lubricator 4 weeks (8 weeks from baseline).

\section{Participants}

22 dependent elderly people were recruited from two places, the Department of Dental medicine at the unit for Gerodontics at Karolinska institutet, and from Public Dental Care at the unit for Medical dentistry at Stockholms sjukhem. The inclusion criteria were participants over 65 years, with expressed dry mouth symptoms, xerostomia, and understanding Swedish. The exclusion criteria were cognitive disorders as diagnosis of dementia or other limitations that were obstacles for performing the study activities as functional limitations from stroke and neurological diseases. An application for ethical approval was obtained by the Regional ethics committee in Stockholm. The study was classified as a product test 2012/597-31/4. The participants signed an informed consent about the study. They could discontinue their participation without giving any reason.

\section{Study process and products}

The participants started to use one of the two test products for four weeks and after that the other product for four weeks, a modified cross-over design. After each study measurements were carried out and added to the study protocol, ending up in three measurement sessions for each individual. The products were Proxident oral spray with sunflower oil, and Proxident gel-based flavorless oral moisturizer. The products are made in Sweden by Proxident AB. All examinations were carried out by the same two persons (author AG and CC) together mostly in the participants own home with a mirror, probe and flash light. Before the start of the study the participants or the care staff got instructions on how to use the products and receive new bottles and how they could assist the elderly. A protocol with instructions for how to use the product as often they needed and always before bedtime was posted in the patients' bathroom together with a self-reported note list.

\section{Data collection}

The study started with baseline data, medications, general health, dental status, gingival and plaque index, mucosal plaque score, objective measures of salivation and supra gingival plaque samples for microbial analyses. In addition to the clinical data, two formulas for patient evaluation were used, OHIP-14 and VAS-scales.

\section{Clinical and microbial data}

The condition of gingiva was registered by Gi (9) from 0-3.0 healthy gingiva, 1 slight inflammation, 2 moderate inflammation, 3 heavy inflammation. Plaque was registered with $\mathrm{Pi}(10)$ from $0-3,0$ no visible plaque, 1 slight plaque registered with a probe, 2 visible but thin plaque on some or all tooth surfaces, 3 visible thick plaque on almost all or all surfaces. Mucosal plaque score, MPS, is used for both persons with and without natural teeth and prostheses. The index was designed to evaluate oral health and oral hygiene in groups of individuals at institutions and in hospitals. It is composed by two measurements, MS for mucosal disorders, criteria (1) Normal gingiva and mucosa (2) Mild inflammation (3) Moderate inflammation (4) Severe inflammation and PS plaque score (1) No easily visible plaque (2) Small amounts of plaque (3) Moderate amounts of plaque (4) Abundant amounts of confluent plaque. They are added to a single index, measuring a persons' oral hygiene, not in order to make a diagnosis. ${ }^{11}$ The participants rested 15 minutes after arrival, the time was registered and they had not eaten, smoked or brushed their teeth for one hour before collecting saliva for a 15 minute period. The participants bend forward and drool in a cup and then the amount of saliva was measured. After that they chewed a piece of paraffin for 30 seconds and then collected stimulated saliva for 5 minutes which was measured. ${ }^{12}$ The saliva collections were mainly performed in the morning time. Supra-gingival plaque was collected with sterile pellets, from four interproximal supra-gingival sites. The sampling sites were between the upper right first and second molars, the lower left first and second molars, the upper right second incisor and the canine and the lower left second incisor and the canine. If one or more of these sites were not available, the closest available site was selected. The samples were transferred to transport medium VMGA III and processed within 24 hours. The analysis of the samples has previously been described [13]. The bottles with VMGA III were shaken on a whirly mixer for 10 seconds and thereafter 0.1 $\mathrm{ml}$ was placed in a standardized fashion on each agar plate, using unselective enriched blood agar plates and selective agar plates. The total number of microorganisms growing under anaerobic conditions, colony-forming units (CFU) and the proportion of Candida albicans, Staphylococcus aureus, enterococci, Streptococcus mutans, lactobacilli and pseudomonas were calculated. Semi quantitative estimations were made according to the following scale: Very sparse growth: 1-10 CFU

Sparse growth: 11-100 CFU

Moderate growth: 101-1.000 CFU

Heavy growth 1.001-10.000 CFU

Very heavy growth $>10.000$ CFU. ${ }^{14}$

The agar plates were made at the laboratory with material from Oxid Ltd (Basingstoke, Hampshire, UK) and Difco Laboratories, Becton, Dickinson and Company (Sparks, MD, USA). We created four groups to take account of the bacteria in this fashion:

1. No growth

2. Very sparse growth and sparse growth=Rare 
3. Moderate growth and heavy growth=Moderate

4. Very heavy growth=Abundant

\section{Patient evaluation}

Participants rated their own oral health according to OHIP-14 and VAS scales. OHIP-14 provides an indication of what kind of symptoms the patient suffers from and the VAS scales gives information about how the symptoms changed over the study period with the last 4 weeks as a reference. OHIP-14 is a shortened version of the Oral Health Impact Profile, which is a standardized and validated form, originally composed of 49 items [15]. OHIP looks at 7 different dimensions; functional limitation, physical pain, psychological discomfort, physical disability, psychological disability, social disability and handicap. It is graded on a 5-point scale besides an answer that the question is not applicable for the actual person and situation. Visual Analogue Scales (VAS) can be used in the diagnosis of salivary flow dysfunction and to detect changes in saliva flow values over time [16]. The questionnaire consists of 8 claims where the patient estimates their discomforts linked to dry mouth. The scale ranges from $0-100$ $\mathrm{mm}$ with the estimation "very much" at $0 \mathrm{~mm}$ and "not at all" at 100 $\mathrm{mm}$. Participants were asked to the mark their opinion with an $\mathrm{X}$ on the line.

\section{Statistical analysis}

All collected data was registered in an Excel file and the Statistical Sciences of Social Package 16.0 (SPSS) was used. Paired ttest was used for analysis of possible differences between the treatment periods. Chi2 was used for salivary flow, MPS, GI and PI. The data from OHIP-14 was dichotomised and the VAS-scales were divided into three groups "very", "moderate" and "not at all". McNemar test and Bowker's test for symmetry and Friedman ANOVA was used to compare measurements in the same person concerning microbial growth. P-values $\leq 0.05$ were considered statistically significant.

\section{Results}

In this study we had a baseline of 22 participants. Six of them quit after baseline, and one did only test the oil product. Two persons felt they did not need the products, one had private reasons and one was in hospital, three others did not give any reason. See Figure 1. Of the 15 participants that followed the whole study, there were 12 women and three men, born 1924-49, 6 living in a nursing home and 9 on their own. They had an average of 19 natural teeth and 4 of them had prosthesis. According to self-reported information, they suffered from dry mouth daytime and/or night-time. The study products were used on average four times every twenty-four-hour. No participants used the products less than three times every twenty-four-hours.

\section{State of general health and medicines}

None of the participants had been diagnosed with diabetes but $33.3 \%(n=5)$ suffered from cardiovascular diseases and $13.3 \%(n=2)$ have had pneumonia under the last year. Three persons took no drugs at all but the other took in average 7 drugs, every person more than four drugs each. Most common was Paracetamol 53.3\% ( $\mathrm{n}=8)$ used daily, diuretics $33.3 \%(n=5)$ and sedatives $26.7 \%(n=4)$. The drug intake did not change during the study period

\section{Oral health}

The mean value of MPS decreased from 3.9 to 3.6 after using the oral spray. After using gel-based oral moisturizer there was a further decrease of the mean value to $3.2(\mathrm{p}=0.04)$. The gingival index decreased from 1.1 to $0.7(\mathrm{p}=0.01)$ after using oral spray. After gelbased oral moisturizer the mean value increased to 0.8 which was still an improvement compared to baseline $(\mathrm{p}=0.05)$. Plaque index was nearly unchanged. See Figure 2.

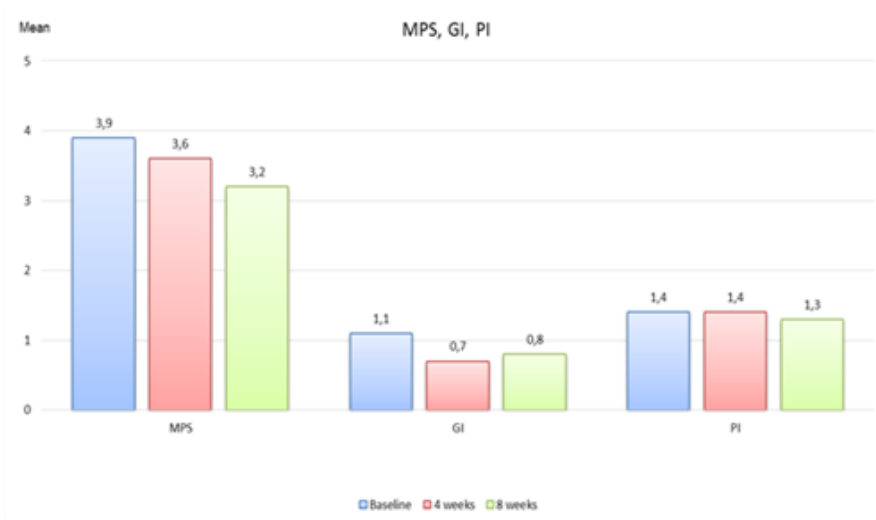

Figure 2 Mean value of MPS (mucosal plaque score), gingival index and Plaque index.

\section{Saliva}

The stimulated saliva showed a decrease after four weeks from $0.87 \mathrm{ml} / \mathrm{min}$ to $0.56 \mathrm{ml} / \mathrm{min}$, then an increase to $0.77 \mathrm{ml} / \mathrm{min}$. The unstimulated saliva was unchanged from baseline to four weeks and then a small increase occurred from $0.04 \mathrm{ml} / \mathrm{min}$ to $0.06 \mathrm{ml} / \mathrm{min}$. See Figure 3.

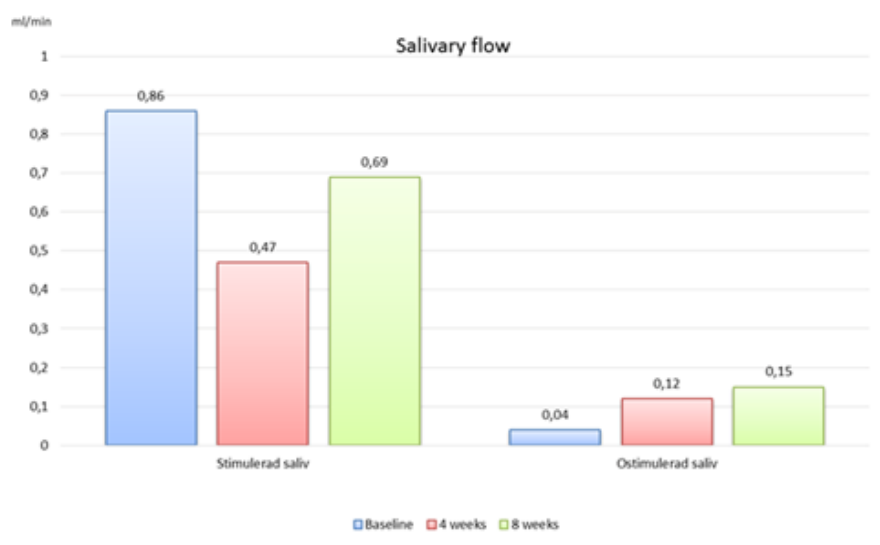

Figure 3 Mean values for salivation $\mathrm{ml}$ per minute.

\section{Microbial results}

At baseline most participants had lactobacillus $(\mathrm{n}=14)$, Candida albicans $(\mathrm{n}=10)$ and Streptococcus mutans $(\mathrm{n}=8)$, two have Staphylococcus aureus and one pseudomonas. This composition of the oral flora was not changed after the 8 weeks study period but according to Candida albicans, there was a change between the groups during the study period. Some of the participants migrated 
from moderate to the rarely group, but the change was not statistically significant, see Figure 4.

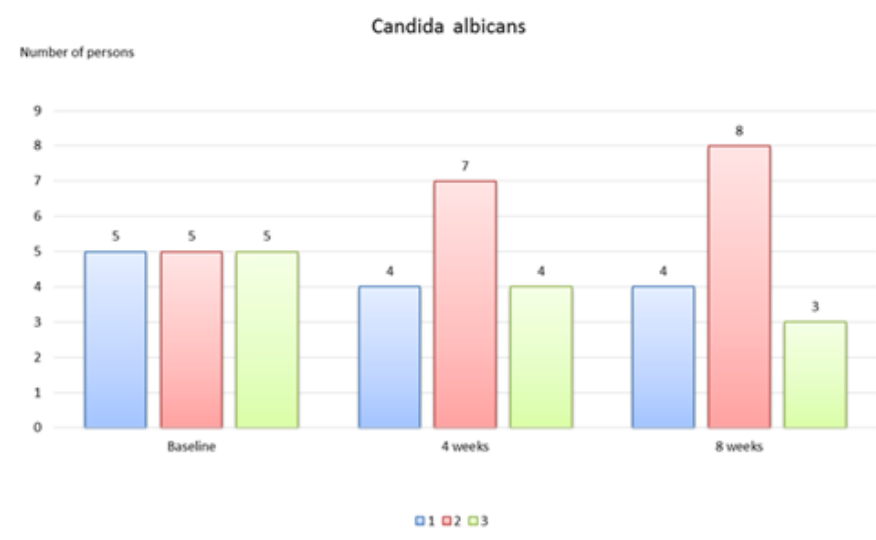

Figure 4 Candida albicans I: No growth; 2: Rarely; 3: Moderate Both after using spray and gel Candida albicans were placed in lower growth groups; $p=0.9536$ from baseline- 4 weeks (spray) and $p=0.8013$ from baseline- 8 weeks(gel).

\section{Patient evaluation}

OHIP-14: At baseline $29 \%(\mathrm{n}=4)$ experienced difficulties with eating and that they had interrupted their meals. After the use of gel $80 \%(\mathrm{n}=12)$ reported that they did not need to interrupt their meals $(\mathrm{p}=0.01)$. At baseline $43 \%(\mathrm{n}=6)$ had difficulties to relax and after 4 weeks of spray, $21 \%(n=3)$ claimed this and after 4 weeks with gel, $13 \%(\mathrm{n}=2)$ answered that they often experienced this $(\mathrm{p}=0.04)$. Then also the feeling of irritation in other people had decreased $(\mathrm{p}=0.04)$. See Table 1.

VAS scales: $79 \%(n=11)$ described that they had a very dry mouth at baseline. The question "How much saliva is in your mouth" showed an improvement between 4 and 8 weeks $(\mathrm{p}=0.01)$. At baseline experienced $43 \%(n=6)$ that they had very difficult to talk and after 8 weeks this problem had decreased to $29 \%(n=4)(p=0.04)$. After the spray period $71 \%(\mathrm{n}=10)$ felt very dry in their mouths and after gel $60 \%(n=9)$. The question" Rate the level of your thirst" showed significant improvement $(\mathrm{p}=0.01)$ after 4 weeks with spray. $71 \%$ $(\mathrm{n}=10)$ described at baseline that they felt themselves as very thirsty and after 4 and 8 weeks $43 \%(n=6)$ and $53 \%(n=8)$ respectively reported this. See Table 2.

Table I Numbers of respondents that agrees with claim, OHIP-I4

\begin{tabular}{lllll}
\hline & & Baseline-4 Weeks & 4-8 Weeks & Baseline-8 Weeks \\
\hline Trouble pronouncing words & $\mathrm{n}$ & $\mathrm{II}$ & $\mathrm{II}$ & 10 \\
Sense of taste has worse & $\mathrm{n}$ & 9 & 9 & 7 \\
Painful aching in the mouth & $\mathrm{n}$ & $9(\mathrm{p}=0.01 \varphi)$ & 7 & 7 \\
Uncomfortable to eat food & $\mathrm{n}$ & 5 & 9 & $8(\mathrm{p}=0.01 \mathrm{l})$ \\
Been self-conscious & $\mathrm{n}$ & 7 & 7 & 6 \\
Felt tense & $\mathrm{n}$ & 9 & 10 & 8 \\
Diet been unsatisfactory & $\mathrm{n}$ & 7 & 9 & 6 \\
Had to interrupt meal & $\mathrm{n}$ & 4 & 6 & 3 \\
Difficult to relax & $\mathrm{n}$ & $10(\mathrm{p}=0.04 \varphi)$ & 10 & 8 \\
Been embarrassed & $\mathrm{n}$ & 7 & 7 & 7 \\
Been irritable with others & $\mathrm{n}$ & $8(\mathrm{p}=0.04 *)$ & $10(\mathrm{p}=0.04 \varphi)$ & 9 \\
Difficulty doing usual jobs & $\mathrm{n}$ & 8 & 9 & 7 \\
Felt life less satisfying & $\mathrm{n}$ & 12 & $1 \mathrm{I}$ & 10 \\
Totally unable to function & $\mathrm{n}$ & 3 & 3 & 3 \\
\hline
\end{tabular}

$p \leq 0.05$ was deemed to be significant; $\varphi$ : significant improvement; *: the value deteriorates

Table 2 Numbers of respondents that answered much or pretty much,VAS

\begin{tabular}{|c|c|c|c|c|}
\hline & & Baseline-4 Weeks & 4-8 Weeks & Baseline-8 Weeks \\
\hline Rate the difficulty you experience in speaking due to dryness & $\mathrm{n}$ & 10 & 12 & $13(p=0.04 \varphi)$ \\
\hline Rate the difficulty you experience in swallowing due to dryness & $\mathrm{n}$ & 8 & 8 & 12 \\
\hline Rate how much saliva is in your mouth & $\mathrm{n}$ & 5 & $4(p=0.01 \varphi)$ & 6 \\
\hline Rate the dryness of your mouth & $\mathrm{n}$ & 13 & 13 & 14 \\
\hline Rate the dryness of your throat & $\mathrm{n}$ & $10(p=0.04 *)$ & 11 & 12 \\
\hline Rate the dryness of your lips & $\mathrm{n}$ & 11 & 9 & 10 \\
\hline Rate the dryness of your tongue & $\mathrm{n}$ & 9 & 10 & 11 \\
\hline Rate the level of your thirst & $\mathrm{n}$ & II $(p=0.01 \varphi)$ & 10 & 11 \\
\hline
\end{tabular}

$\mathrm{p} \leq 0.05$ was deemed to be significant; $\varphi$ : significant improvement; *: the value deteriorates 


\section{Discussion}

\section{Method discussion}

Due to the small amount of participants and a study design that was meant to sort suitable variables for following studies, we consider the present study as a pilot. In this pilot study we were restricted to a small study group in rather frail health condition. It was problematic both to encourage the participants to follow the whole study period and to come to the scheduled dental clinic visits. ${ }^{17}$ If a larger study with this focus is planned, it is probably better to recruit participants from, for example, a general practitioner clinic. We also treated the population as one group, even if there were some changes in their age, medical conditions and drug use. It was not suitable to divide the group into small subgroups and the participants served as their own controls. We included study participants based on the inclusion criteria dry mouth and more than 65 years old. None of the participants was smoking, yet smoking is associated with dry mouth. ${ }^{18}$ Saliva samples were all taken during the morning session but not at the exact time which is a weakness. Further on, bacteria such as Streptococcus mutans, lactobacilli and Candida albicans may require prolonged use of products for possible changes. The bacteria samples could also have been analyzed in a different way, with a more specific measurement of numbers and frequencies of bacteria. The product Oral spray with sunflower oil is commonly used at hospitals and in nursing homes in many years and the other product gel-based flavorless oral moisturizer was a newly introduced product we wanted to evaluate. Quality of life is complex and difficult to measure. There are instruments aimed to measure the general quality of life such as General Health Questionnaire $(\mathrm{GHQ})^{19}$ but there are also questionnaires designed to measure oral health related quality of life, where the relationship between oral health and quality of life are highlighted. ${ }^{20}$ OHIP-14 is adapted for elderly and was therefore chosen to be used in this study.

\section{Saliva flow}

Plaque index was constant during the study time; a weak decrease could be seen after 8 weeks but was not statistically significant. However, the improved values for gingival index GI and MPS suggest that the oral mucosal conditions improved. Unstimulated saliva increased after 8 weeks but no statistical significance could be shown here. Concerning stimulated saliva a decrease was observed after 4 weeks and then an increase again after 8 weeks. The amount of saliva produced is influenced by several factors; the "biological clock", the time of day, stress, chewing power and number of teeth. ${ }^{12}$ Several participants in the study said they were troubled by dry mouth mostly at night. We intended to perform the saliva measurements at the same time of day but had to make some alterations in the time schedule that can have influenced the result. Possibly sympathetic innervation also must be taken into account. It may have influenced the results when participants were nervous of the first test and then become more comfortable. ${ }^{21}$ Salivary glands are affected both by sympathetic and parasympathetic innervation, therefore resting saliva and stimulated saliva varies. ${ }^{22}$ It is mostly the mucus-resting saliva which influence if you experience dry mouth. The attendees were elderly people over 65 years old with an average of 6.9 different medications on a daily basis. More than four drugs daily contribute to a risk of suffering from dry mouth. ${ }^{23}$ Many seniors take several medicines with oral dryness as a co-effect. ${ }^{22}$

\section{Oral health}

Participants had maintained their oral hygiene habits and the slight rise of saliva was not big enough to affect the amount of plaque. The plaque index was relatively constant during the study time. Dry mouth increases the plaque of the oral cavity which leads to gingivitis. ${ }^{24} \mathrm{An}$ improvement of gingival index could be seen after 4 weeks when oral spray with sunflower oil was used. The index went up slightly after the use of mouth moisture gel but the value was lower than at the baseline indicating that the products have a positive impact on gingiva. The MPS is a unified index for amount of plaque and oral mucosal condition, both for dentate and denture wearers. ${ }^{12}$ MPS showed an average decrease during the study time. Decreased mucus saliva makes the oral mucosa brittle and causes great discomfort for the patient. ${ }^{25}$ The unstimulated saliva is harder to substitute and saliva replacement products can be recommended frequently to dry mouth patients to good effect. Stimulated saliva can be impacted by the individual by the numbers and consumption of food and drink. ${ }^{4}$

\section{Micro-flora}

Streptococcus mutans was constant but lactobacilli increased slightly. Many of the participants had sweets and other sugar products to hand and it is known that at low salivary secretion rates, the $\mathrm{pH}$ of saliva and buffer capacity decreases. ${ }^{26}$ The evaluated products did not seem to have the capacity to change these circumstances during the study period. In Candida albicans the participants move from a higher group to a lower one. Candida albicans was a frequent finding in our study group but it decreased over time depending on increased oral hygiene or possible effect from the products. However, this finding should be regarded as a trend, since statistical significance was not reached.

\section{Patient evaluation}

Today there is no treatment for dry mouth, since it is largely caused by medical drug use and diseases. ${ }^{27}$ Elderly are often associated with the largest oral problems and suffer from dry mouth to a greater extent than the rest of the population ${ }^{28}$ Saliva production decreases with ageing and women produce less saliva then men. It may be due to increased medication with age, but also that women tend to take more medications than men. ${ }^{29}$ In this study all participant were elderly and the group consisted of more women than men. It may have been more women who expressed an interest in participating as they experience more discomfort with dry mouth. Many studies point to the association between xerostomia and quality of life..$^{27}$ Saliva is an important factor to function in everyday life and to be able to interact with other people. ${ }^{30}$ There are few studies about products that are recommended in oral dryness. They are often not registered as medicines and review articles have concluded that data are limited and sometime ambiguous. ${ }^{8}$ There are no clear directives about what products should be recommended. ${ }^{31}$

\section{Conclusion}

Proxident oral spray with sunflower oil and moisture gel without taste may help patients suffering from dry mouth to improve their oral mucous conditions, but it requires further studies to confirm these results. The products can be recommended as comfort products.

\section{Funding}

None.

\section{Acknowledgments}

Thanks to Elisabeth Berg KI, LIME/MedStat for processing statistical data, Maude Wikström, Sahlgrenska academy for microbial advices and Patricia De Palma for help with diagram. 


\section{Conflicts of interest}

The study was funded by grants from Konung Gustaf V:s och Drottning Victorias Frimurarestift else and Proxident AB supplied the products.

\section{References}

1. Gupta A, Epstein JB, Sroussi H Hyposalivation in elderly patients. J Can Dent Assoc. 72:841-846.

2. Wimmer BC, Dent E, Visvanathan R, Wiese MD, Johnell K, et al. Polypharmacy and medication regimen complexity as factors associated with hospital discharge destination among older people: a prospective cohort study. Drugs Aging. 2014;31(8):623-630.

3. Porter SR, Scully C, Hegarty AM. An update of the etiology and management of xerostomia. Oral Surg Oral Med Oral Pathol Oral Radiol Endod. 2004;97(1):28-46.

4. Nederfors T, Henricsson V, Dahlöf C, Axéll T. Oral mucosal friction and subjective perception of dry mouth in relation to salivary secretion. Scand $J$ Dent Res. 1993;101(1):44-48.

5. Scannapieco FA. Pneumonia in nonambulatory patients. The role of ora bacteria and oral hygiene. J Am Dent Assoc. 2006:137 Suppl: 21S-25S.

6. Pace CC, Mc Cullough GH The Association Between Oral Microorganisms and Aspiration Pneumonia in the Institutionalized Elderly: Review and Recommendations. Dysphagia. 2010;25(4):307-322.

7. Quagliarello V, Ginter S, Han L, et al. Modifiable Risk Factors for Nursing Home-Acquired Pneumonia. Clin Infect Dis. 2005;40(1):1-6.

8. Ota Y, Morito A, Fujisawa K, et al. Evaluation of a moisturising microgel spray for prevention of cell dryness in oral mucosal cells: an in vitro study and evaluation in a clinical setting. Eur J Cancer Care (Engl). 2012;21(6):728-734.

9. Löe H, Silness J. Periodontal disease in pregnancy. I. Prevalence and severity. Acta Odontol Scand. 1963;21: 533-551.

10. Silness J, Löe H. Periodontal disease in pregnancy. II. Correlation between oral hygiene and periodontal condition. Acta Odontol Scand. 1964;22: 121-135.

11. Henriksen BM, Ambjørnsen E, Axéll TE Evaluation of a mucosal-plaque index (MPS) designed to assess oral care in groups of elderly. Spec Care Dentist. 1999;19(4):154-157.

12. Flink $H$, Tegelberg $A$, Lagerlöf $F$ Influence of the time of measurement of unstimulated human whole saliva on the diagnosis of hyposalivation. Arch Oral Biol. 2005;50(6):553-559.

13. Dalén G, Linde A, Möller A, et al. A retrospective study of microbiologic samples from oral mucosal lesions. Oral medicine. 1982;53(3):250-255.

14. Dahlén G. Microbiological diagnosis in oral diseases. Acta Odontol Scand. 2006;64(3):164-168.

15. Slade GD. Derivation and validation of a short-form oral health impact profile. Community Dent Oral Epidemiol. 1997;25(4): 284-290.
16. Pai S, Ghezzi EM, Ship JA. Development of a Visual Analogue Scale questionnaire for subjective assessment of salivary dysfunction. Oral Surg Oral Med Oral Pathol Oral Radiol Endod. 2001;91(3):311-316.

17. Muszalik M, Kornatowski T, Zielińska-Więczkowska H, et al. Functional assessment of geriatric patients in regard to health-related quality of life. Clin Interv Aging. 2014;10:61-67.

18. Dyasanoor S, Saddu SC. Association of Xerostomia and Assessment of Salivary Flow Using Modified Schirmer Test among Smokers and Healthy Individuals: A Preliminutesary Study. J Clin Diagn Res. 2014;8(1): 211 213.

19. Smith B, Baysan A, Fenlon M. Association between Oral Health Impact Profile and General Health scores for patients seeking dental implants. $J$ Dent. 2009;37(5):357-359.

20. Baker SR, Pankhurst CL, Robinson PG. Utility of two oral health-related quality-of-life measures in patients with xerostomia. Community Dent Oral Epidemiol. 2006;34(5):351-362.

21. Clark CE, Horvath IA, Taylor RS, et al. Doctors record higher blood pressures than nurses: systematic review and meta-analysis. $\mathrm{Br} \mathrm{J} \mathrm{Gen}$ Pract. 2014;64(621):e223-e232.

22. Nederfors T. Xerostomia and hyposalivation. Adv Dent Res. 2000;14:48 56.

23. Wårdh I,Wikstöm M. Long-term effects of using oral care aides at a nursing home for elderly dependent residents-a pilot study. Spec Care Dentist. 2014;34(2):64-69.

24. Gerdin EW, Einarson S, Jonsson M, et al. Impact of drymouth conditions on oral health-related quality of life in older people. Gerodontology. 2005;22(4):219-226.

25. Cassolato SF, Turnbull RS. Xerostomia: clinical aspects and treatment. Gerodontology. 2003;20(2):64-77.

26. Almståhl A, Wikström M, Kroneld U. Microflora in oral ecosystems in primary Sjögren's syndrome. J Rheumatol. 2001;28(5): 1007-1013.

27. Friedman B, Heisel M, Delavan R. Validity of the SF-36 five-item Mental Health Index for major depression in functionally impaired, communitydwelling elderly patients. J Am Geriatr Soc. 2005;53(11):1978-1985.

28. Locker D, Matear D, Stephens M, et al. Oral health-related quality of life of a population of medically compromised elderly people. Community Dent Health. 2002;19(2):90-97.

29. Billings RJ, Proskin HM, Moss ME. Xerostomia and associated factors in a community-dwelling adult population. Community Dent Oral Epidemiol. 1996;24(5):312-316.

30. Strassburger C, Kerschbaum T, Heydecke G. Influence of implant and conventional prostheses on satisfaction and quality of life: A literature review. Part 2: Qualitative analysis and evaluation of the studies. Int $J$ Prosthodont. 2006;19(4):339-348.

31. Ship JA, Mc Cutcheon JA, Spivakovsky S, et al. Safety and effectiveness of topical dry mouth products containing olive oil, betaine, and xylitol in reducing xerostomia for polypharmacy-induced dry mouth. J Oral Rehabil. 2007;34(10):724-732. 